\title{
Will Czochralski Growth of Sapphire Once Again Prevail?
}

\author{
F.J. Bruni ${ }^{a *}$, C.-M. LiU $^{b}$ And J. StOnE-SUndBerG ${ }^{c}$ \\ ${ }^{a}$ Consultant, P.O. Box 2413, Santa Rosa, California, 95405, USA \\ ${ }^{b}$ Sino-American Silicon Products Inc., Innovation Technology Research Center, No. 8, Industry E. Rd. II \\ Hsinchu Science Park, Hsinchu 30075, Taiwan, R.O.C. \\ ${ }^{c}$ Current address: Adjunct Research Faculty, Department of Physics, Portland State University \\ P.O. Box 751, Portland, OR 97207-0751, USA
}

\begin{abstract}
In the past decade there has been an explosive growth in the consumption of sapphire driven by the demands of the next generation of energy efficient general lighting based on GaN LEDs. This application requires orienting these rhombohedral corundum crystals such that the substrate surface is the $c$-plane; a basal plane defined using hexagonal axes. Sapphire crystals form a strong facet on the $c$-plane, and growth in that direction generally results in crystals with high defect densities, particularly dislocations, and low angle grain boundaries. To overcome this drawback, the usual methodology is to grow the crystal in the $a$-direction and then core drill rods perpendicularly which are then sliced into $c$-plane substrates. For all crystal growth techniques commonly employed for sapphire, this approach suffers from poor material utilization. Although this has generally been viewed as an acceptable trade-off in the manufacturing process as long as $2^{\prime \prime}$ substrates were the dominant market, as substrate diameters have increased towards $150 \mathrm{~mm}$ and larger, this compromise is no longer seen as a viable alternative because of the low material utilization and the high energy consumption of the growth process. This has led to a renewed look at the Czochralski process for more efficient $c$-axis substrate production.
\end{abstract}

DOI: 10.12693 /APhysPolA.124.213

PACS: 81.10.Fq, 81.10.Aj, 61.72.-y

\section{Introduction}

Sapphire $\left(\alpha-\mathrm{Al}_{2} \mathrm{O}_{3}\right)$ has been in continuous production for nearly 125 years either in its pure form or doped with chromium (ruby). Every major crystal growth technique has been used at one time or another for sapphire crystal growth - Verneuil, Czochralski, Kyropoulos, Bridgman, edge-defined film-fed growth (EFG), heat exchanger method (HEM) and even solution growth [1]. The applicability of sapphire to so many different crystal growth approaches stems from its unique combination of properties including having a congruent melting point, being relatively chemically inert, hard, tough and having a high thermal conductivity (for an oxide ceramic). This makes it compatible with a variety of crucibles (tungsten, molybdenum and iridium), insulation types (zirconia, graphite and refractory metal radiation shields), heating methods (induction, resistance heating with either refractory metals or graphite, and even gas torches as in the Verneuil method) and furnace ambients (vacuum, inert gas and oxidizing atmospheres).

Sapphire's unique combination of chemical, mechanical, electrical and optical properties make it suitable for a wide range of product applications, so many of these crystal growth methodologies remain commercially viable for niche markets as in the case of EFG for its ability to produce near net shape sapphire parts. The dominant sapphire market today is for substrates used in the man-

*corresponding author; e-mail: fjbruni@aol.com ufacture of blue LEDs via the growth of a GaN layer on a sapphire substrate by vapor phase epitaxy. In addition to filling out the color spectrum for LEDs and thus making full-color, solid-state displays practical, GaN blue LEDs can be combined with a phosphor to produce white light. This makes them ideal for backlighting of flat screen displays but it also makes them exactly suited for the general lighting market. The substitution of high-brightness LEDs for incandescent and fluorescent light bulbs is expected to make a significant impact on worldwide energy consumption and its effect on the environment. It is a measure of the complexity of achieving the size, orientation and crystalline quality of sapphire LED substrates that there are no fewer than five different crystal growth processes currently in commercial use [2] and one additional in possible development [3].

Corundum, the crystalline form of alpha-aluminum oxide, has trigonal symmetry and belongs to the $R-3 C$ space group. It is usually described with hexagonal axes when identifying specific axes and planes. The sapphire basal plane (0001) is a low energy surface and forms a facet during crystal growth. To grow a crystal in the $c$-direction, one must deal with facets on the growth interface and the crystalline defects that result. The standard way to overcome this issue for the production of $c$-plane sapphire substrates has been to grow the crystal in the $a$-direction [11 $\overline{2} 0]$ and "core" out rods perpendicular to the growth direction. This has been the method of choice for Czochralski, Kyropoulos and HEM-grown crystals.

As long as wafer diameters were small $\left(2^{\prime \prime}\right.$ is still the dominant market in Taiwan, for example), the 
Czochralski process could compete with others, mainly Kyropoulos. However, as in the silicon industry, processes demanding larger wafer sizes are always being developed. The limitations of iridium crucibles make Czochralski-grown sapphire crystals with diameters much above $150 \mathrm{~mm}$ problematic. Thus, using the standard $a$-axis growth and perpendicular coring technology, they are limited to a maximum product wafer diameter of $100 \mathrm{~mm}$, and, even then, the material utilization is rather poor. More recently, Sumitomo Metal Mining announced the development of the Czochralski growth of $300 \mathrm{~mm}$ diameter $a$-axis sapphire crystals for the production of $150 \mathrm{~mm}$ diameter wafers [4].

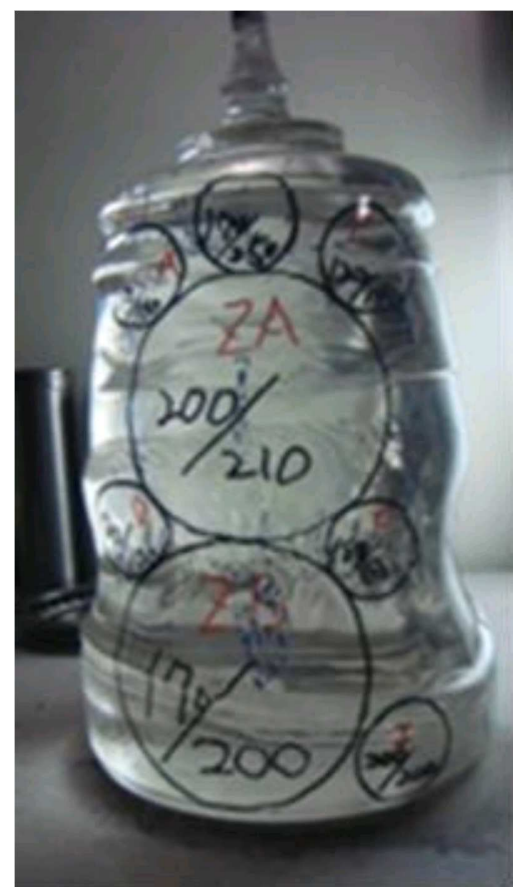

Fig. 1. A $90 \mathrm{~kg}$ Kyropoulos-grown sapphire crystal mapped for core drilling two $6^{\prime \prime}$ rods and several $2^{\prime \prime}$ rods.

The ability of the Kyropoulos process to grow significantly larger crystals has enabled this procedure to largely displace the Czochralski method at the present time. Figure 1 shows a $90 \mathrm{~kg}$ sapphire crystal grown in a Thermal Technology Kyropoulos furnace and "mapped" for two $6^{\prime \prime \dagger}$ cores and several $2^{\prime \prime}$ cores. Figure 2 shows a typical Kyropoulos crystal after coring, ready to be cleaned up and recycled into the growth process. This figure also graphically demonstrates the large volume of crystallized sapphire in the Kyropoulos process that goes unused representing a significant energy investment due

$\dagger$ In the electronics industry substrates smaller than $100 \mathrm{~mm}$ are in English units (inches) and those larger than $3^{\prime \prime}$ are measured in metric units (millimeters). However, it is typical to refer to the metric sizes by their nearest English equivalent.



Fig. 2. A Kyropoulos-grown sapphire crystal after core drilling $2^{\prime \prime}$ rods and ready to be recycled into the growth process along with several broken or rejected rods.

to the high melting point of sapphire as well as the slow growth rate.

Another issue related to the Kyropoulos and HEM growth processes is that the growth rate is difficult to measure. This in turn leads to relatively slow crystallization rates in order to stay below the critical rate that would nucleate defects, a factor which adds to the production cost both in terms of energy consumption and capital hardware.

The crystal grown on the Thermal Technology Kyropoulos system shown in Fig. 1 was controlled using load cells to calculate the growth rate and a feedback control algorithm. However, this can only be used during the first half of the growth process before the crystal interface touches the bottom of the crucible, after which the remainder of the crystal was grown using downward ramping of the power. In general though, Kyropoulos systems do not employ any growth rate measurement or feedback procedures. The Czochralski process on the other hand readily lends itself to precise growth rate control. These factors have led to renewed interest in its use for $c$-axis growth of sapphire.

\section{Bridgman and variants}

The first attempt to commercialize a melt growth process for sapphire along the $c$-axis was the recent combined heat extraction system (CHES) process by ARC Energy. This is a variation on the HEM process with a somewhat more sophisticated method of controlling the growth rate by vertical crucible translation as in the Bridgman method. Crystals as large as $250 \mathrm{~mm}$ diameter have been grown [5]. c-axis growth has been demon- 
strated in a more traditional Bridgman furnace by Miyagawa et al. for crystals up to $76 \mathrm{~mm}$ in diameter [3]. Crystals grown by both of these methods show high dislocation densities and a tendency toward low-angle grain boundaries (LAGB) also known as "lineage".

\section{Czochralski growth}

One of the earliest attempts to grow $c$-axis (or what is sometimes called $0^{\circ}$ ) sapphire was by Belt and Puttbach [6]. They grew $\approx 25 \mathrm{~mm}$ diameter crystals which had very high dislocation densities $\left(10^{4}-10^{5} / \mathrm{cm}^{2}\right)$ and LAGB. Yip and Brandle [7] also observed that sapphire "grown in the $0^{\circ}$ (i.e. [0001]) orientation frequently has an irregular, cascade-like subboundary network on the boule surface". They went on to say that, when examined using a low power microscope, the (0001) plane contained a massive network of subgrain boundaries. It is for this reason that growth of $c$-axis sapphire has been confined to applications such as missile domes where the requirement of the optical axis is more important than defects.

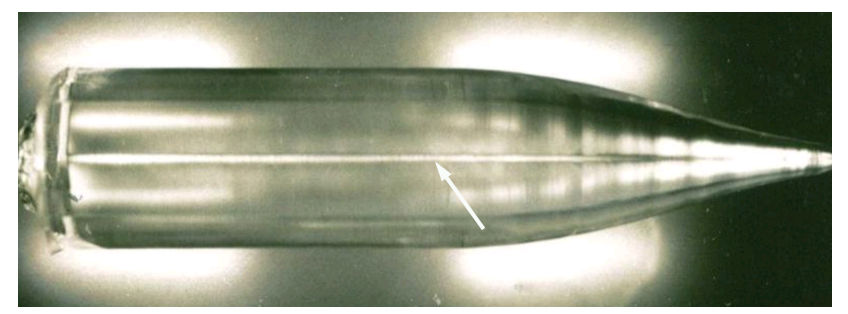

Fig. 3. A neodymium doped yttrium aluminum garnet crystal viewed with polarized light. The bright line down the center (arrow) is the stressed "core" region caused by $\{112\}$ type facets on the growth interface. The angle of the arrow approximates the shape of the growth interface.

Sapphire differs from the garnets such as yttrium aluminum garnet (YAG) and gadolinium gallium garnet (GGG), to cite two examples. The garnets also form facets on the growth interface, and these facets result in a highly strained region in the crystal as shown in Fig. 3. The bright line down the central axis of the crystal is called the "core" and represents unusable material. However, the material grown away from the core region, which crystallizes on an atomically rough surface, has a low defect density and high optical quality. Sapphire, which also grows with a convex growth interface, does not have its defects concentrated in a core region. Rather, the entire crystal cross-section is impacted by defects when grown in the $0^{\circ}$ orientation.

\section{Low angle grain boundaries}

The elimination of low angle grain boundaries in $c$-axis sapphire is the primary challenge. It has been found that, with the proper control of the temperature gradient and the hot zone design as well as with strict limits on the seed lift rate, sizable $c$-axis sapphire crystals

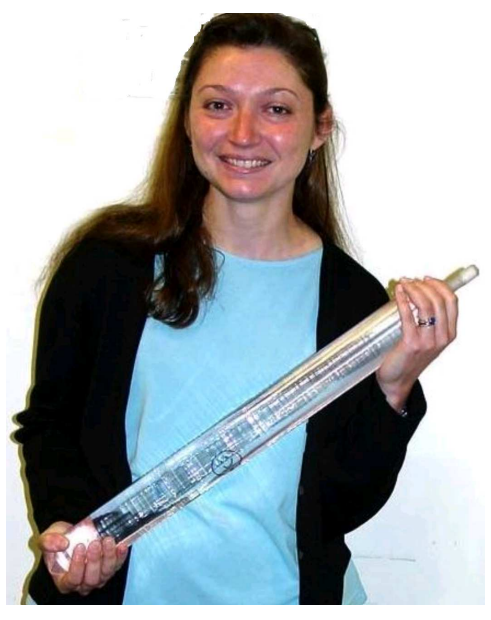

Fig. 4. One of the authors holding a $2^{\prime \prime}$ diameter, $18^{\prime \prime}$ long $c$-axis sapphire crystal grown free of LAGBs.

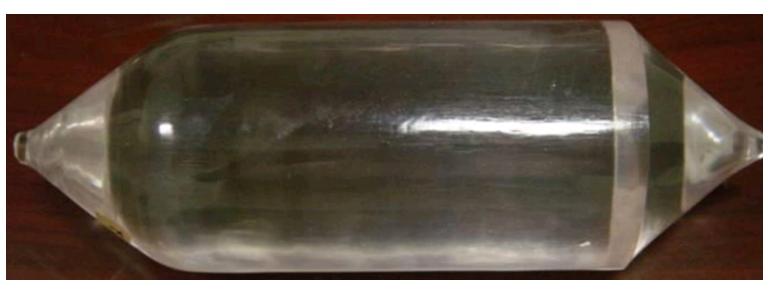

Fig. 5. A $4^{\prime \prime}$ diameter $c$-axis sapphire crystal grown at Sino-American Silicon. The surface texture is an indication of lineage.

can be grown without low angle boundaries (LAGB). Yip and Brandle [7] also pointed out that careful attention to the seeding and necking steps in the Czochralski growth was critical to eliminating low angle grain boundaries. Figure 4 shows one of the authors with a $2^{\prime \prime}$ diameter, $c$-axis sapphire crystal, $18^{\prime \prime}$ long grown with no observable lineage. Figure 5 shows a $4^{\prime \prime}$ diameter $c$-axis sapphire crystal, and Fig. 6 is a detailed view of the same crystal through crossed polarizers showing the clearly evident

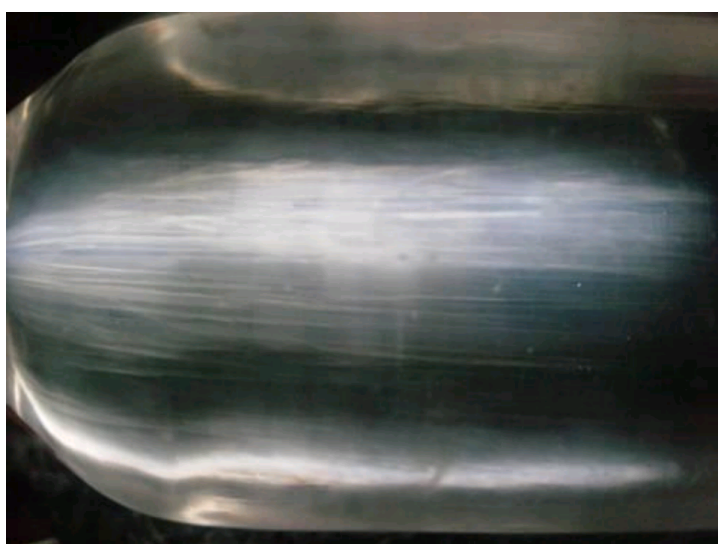

Fig. 6. View of a $4^{\prime \prime} c$-axis sapphire crystal with polarized light showing the lineage of LAGBs. 


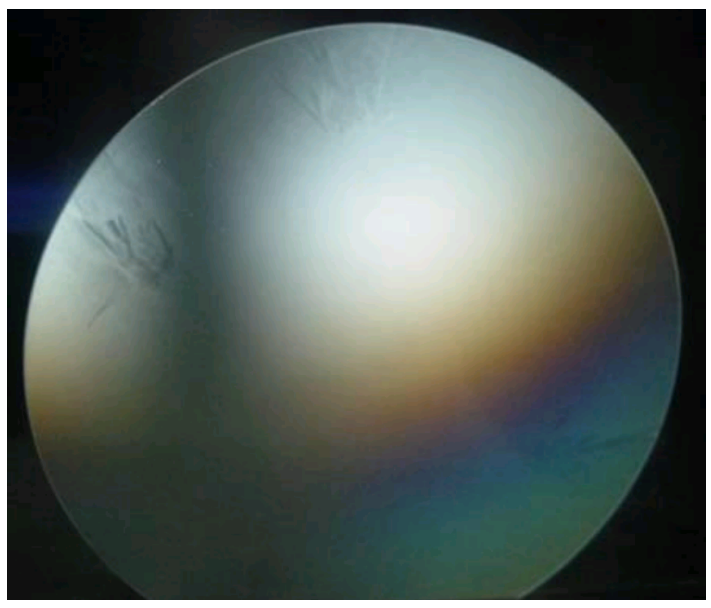

Fig. 7. View of a $4^{\prime \prime} c$-axis sapphire wafer with polarized light showing the lineage of LAGBs.

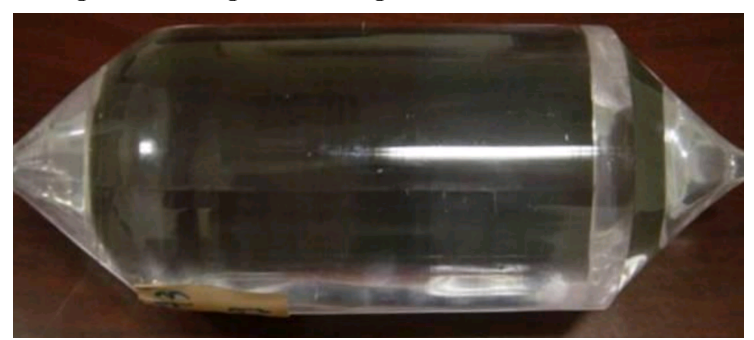

Fig. 8. A $4^{\prime \prime} c$-axis sapphire crystal grown successfully without LAGBs. Note the smoother exterior surface.

LAGBs. Figure 7 shows a wafer sliced from this crystal, again with the LAGBs evident. Figures 8 and 9 show another $4^{\prime \prime} c$-axis boule and wafer without LAGBs. These crystals were grown at Sino-American Silicon. Thus it has been demonstrated that the problem of low angle grain boundaries can be overcome in the Czochralski process with careful attention to the growth conditions.

\section{Dislocations}

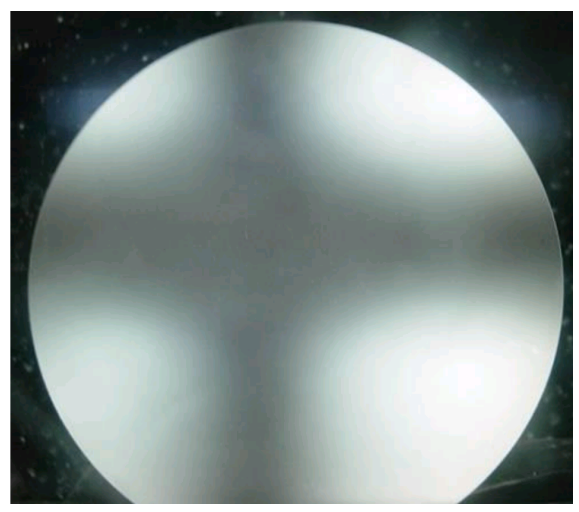

Fig. 9. A polished wafer cut from the crystal shown in Fig. 8 viewed in polarized light and exhibiting no LAGBs.
While there is no doubt that sapphire crystals grown along the $c$-axis have a much higher dislocation density than $a$-axis material regardless of the growth process employed, perhaps by at least an order of magnitude, there is reason to believe that this is not a severe impediment to their use as a substrate for GaN epilayers. Due to the significant $(\approx 16 \%)$ lattice parameter mismatch between sapphire and GaN [8, 9], even epilayers grown on a dislocation-free substrate will have as many as $10^{9}$ threading dislocations per square centimeter [10]. Several techniques have been developed to mitigate this issue. One is to grow a buffer layer of either AlN or GaN on the substrate that serves to block dislocation propagation into the active device layer [11, 12]. Another is to cut patterns into the sapphire surface so that islands of GaN are nucleated on sapphire "mesas" and then grow laterally to form a continuous layer of GaN [8]. The pattern can be comprised of various forms including grooves or dots.

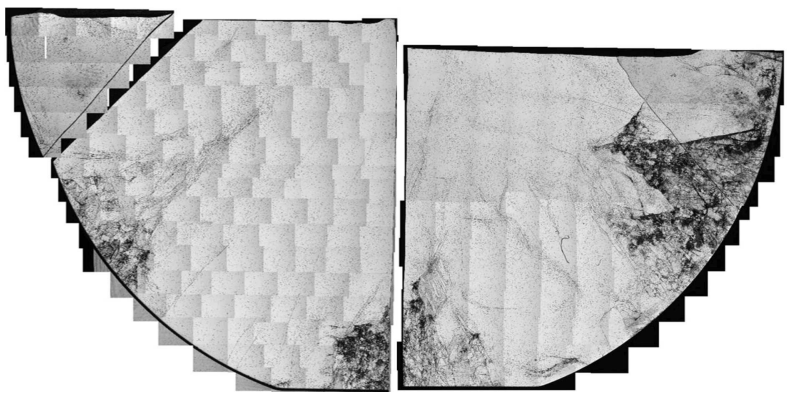

Fig. 10. A mosaic of photos of an etched $c$-axis grown wafer showing how dislocations (indicated by etch pits) are focused into regions along the $a$-axes.

During the Czochralski crystal growth, when a dislocation propagates from one layer of crystal to the next, it assumes the shape of lowest energy which would be the shortest length, assuming no variation of energy with crystallographic orientation. The shortest length would be a straight line perpendicular to the growth interface. However, if the dislocation energy varies with crystallographic orientation, dislocations propagate at an angle to the growth interface and will be "focused" into particular zones in the crystal. This can be seen in Fig. 10 which shows dislocations in a wafer from a $c$-axis grown crystal which are preferentially propagating towards the $a$-planes $\{11 \overline{2} 0\}$. Yet, even with the high dislocation densities seen here it is likely that they can be overcome with the processing techniques described above. However, buffer layers and patterned substrates are not a solution for LAGB.

\section{Process economics}

While the previous discussion has centered on the physical capabilities of the Czochralski process to produce crystals of adequate quality, the question of process economics must be addressed. This aspect of the problem focuses on several issues: crucible material, heating 
methodology, growth speed and furnace design. Traditionally, Czochralski-grown sapphire has required iridium crucibles and induction heating. Iridium is a costly precious metal, and, while some iridium is lost to oxidation which can usually be recovered, the major concern for a large scale production process is the large amount of capital tied up in crucibles. There is another issue, however, that would limit the feasibility of the use of iridium crucibles for large sapphire crystals. Melt growth processes required a temperature gradient to define the position of the solid-liquid interface. As the geometry scales to larger dimensions, if the gradient is maintained at the same value, one ultimately encounters a situation where the temperature at the crucible wall exceeds the melting point of iridium $\left(2446^{\circ} \mathrm{C}\right)$ which is only $400{ }^{\circ} \mathrm{C}$ degrees higher than the melting point of sapphire. At that point, no further scaling of the process is possible. The issues of metal costs and melting point can be resolved through the use of molybdenum $\left(T_{\mathrm{m}}=2623^{\circ} \mathrm{C}\right)$ or tungsten $\left(T_{\mathrm{m}}=3422{ }^{\circ} \mathrm{C}\right)$ crucibles. Although, unlike iridium, molybdenum and tungsten lose much of their value as scrap, a sufficient number of runs can be obtained from each crucible that the cost can easily be amortized to an acceptable value. Tungsten crucibles are routinely used in the Kyropoulos method, and molybdenum is the crucible material generally employed for HEM. Typically, a molybdenum crucible survives for only one crystal growth cycle in the HEM process.

Heating methodology must also be considered. Traditionally, iridium crucibles used to grow sapphire are heated inductively. However, resistance heating is generally more efficient, and graphite heating elements are a cheap and reliable way of providing heat to a high temperature furnace. The Kyropoulos process is generally heated using refractory metal (tungsten) heating elements in a vacuum chamber while the HEM process uses graphite heaters in an argon atmosphere. The crystals shown in Figs. 5 and 8 were grown in a Czochralski system using graphite heaters and molybdenum crucibles.

Perhaps the biggest advantage that the Czochralski process has relative to competing methodologies is the precision of growth rate control. The growth rate of the crystal can be exactly determined by the seed lift rate and the induced melt drop rate and can be controlled with great precision using several feedback measurements such as crystal weight. Neither the Kyropoulos nor HEM processes lend themselves readily to growth rate control which contributes to their long cycle times (typically $\approx 18$ days to produce a crystal of $90-100 \mathrm{~kg}$ ). However, as mentioned above, the crystals shown in Figs. 1 and 2 were grown on a Kyropoulos system that uses load cells to control the growth rate for at least the first half of the crystallization process [2].

The use of graphite insulation versus oxide insulation contributes to a substantial reduction in the cost of energy in a Czochralski system. The authors estimate a cycle time of about 13 days for a $6^{\prime \prime}$ diameter Czochralski sapphire crystal with a net "length at diameter" of
16 inches (equivalent to the $6^{\prime \prime}$ core yield of the $\mathrm{Ky}$ ropoulos crystal shown in Fig. 1). We also estimate that, at diameters of $6^{\prime \prime}$ and larger, Czochralski growth overtakes the Kyropoulos growth in terms of energy required for crystallization, even when taking into consideration the energy cost of post growth annealing. Support for this argument was found with industry expert and analyst Eric Virey who commented that $0^{\circ}$, on-axis growth (Czochralski or otherwise) starts making economic sense at $6^{\prime \prime}$ wafer diameters and above, depending upon growth speed and material yields (E. Virey, private communication, June 18, 2013).

\section{Thermodynamics}

Although the use of molybdenum crucibles, graphite resistance heaters and graphite insulation combine to make a Czochralski system that is more economical than a Kyropoulos system for the growth of large sapphire crystals, the thermodynamic issues cannot be ignored. Figure 11 is an Ellingham [13] diagram of the key components in such a system. This diagram uses data from Reed [14] and shows oxygen partial pressure scales, $\mathrm{H}_{2} / \mathrm{H}_{2} \mathrm{O}$ and $\mathrm{CO} / \mathrm{CO}_{2}$ ratios after Richardson and Jeffes [15].

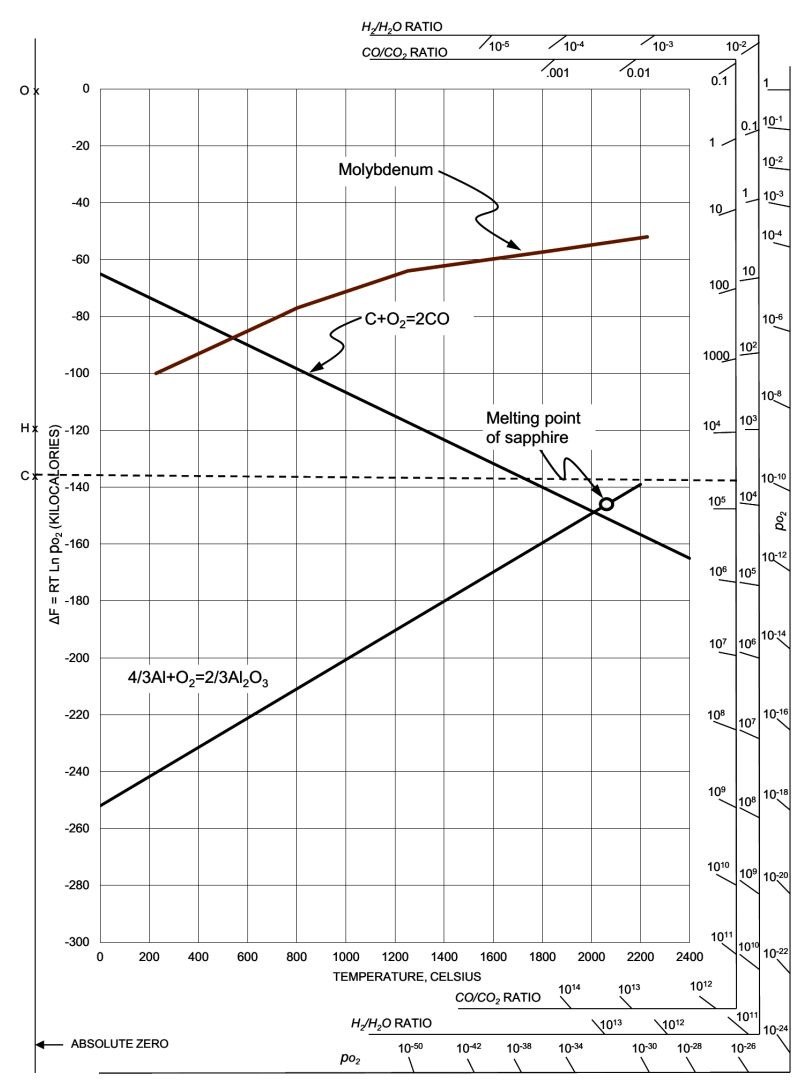

Fig. 11. An Ellingham diagram showing the equilibria with oxygen for the various components of a molybdenum-graphite-sapphire system.

The line labeled $\mathrm{C}-\mathrm{CO}$ represents the free energy as a function of temperature for the lowest oxidation state 
of carbon. Similarly, the line labeled $4 / 3 \mathrm{Al}_{2} \mathrm{O}_{3}+\mathrm{O}_{2}=$ $2 / 3 \mathrm{Al}_{2} \mathrm{O}_{3}$ shows the free energy change for the reaction that forms alumina (sapphire). In order to stabilize the sapphire phase while not simultaneously oxidizing the graphite components, one must be in an ambient that sets the oxygen partial pressure that is above the line for $\mathrm{Al}-\mathrm{Al}_{2} \mathrm{O}_{3}$ and below the line for $\mathrm{C}-\mathrm{CO}$. Clearly, at the melting point of sapphire, it is impossible to be above the line for the alumina equilibrium while simultaneously being below the line for the carbon-CO equilibrium. So a compromise condition must be established. The dotted line represents the oxygen partial pressure resulting from a $\mathrm{CO} / \mathrm{CO}_{2}$ ratio of $\approx 5 \times 10^{4}$. Setting the $\mathrm{CO} / \mathrm{CO}_{2}$ ratio at this level will provide an ambient atmosphere that is oxidizing with respect to alumina and reducing with respect to carbon for all graphite components below $\approx 1700^{\circ} \mathrm{C}$. Graphite components above that temperature would find themselves in a slightly oxidizing atmosphere and would be consumed over time. This compromise may be required in order to grow sapphire crystals of sufficient quality for LED substrates in a graphite-heated system. Growth in a tungsten-heated, vacuum system would not have this limitation.

\section{Conclusions}

The market for sapphire wafers is projected to be dominated by $6^{\prime \prime}$ wafers (rising from $27 \%$ in 2014 to $70 \%$ in 2019 ) while $2^{\prime \prime}$ wafers will disappear by 2020 , and $8^{\prime \prime}$ wafers will represent $20 \%$ of the market by then [16]. By that time, Kyropoulos- and HEM-grown crystals will face the same issues that have plagued Czochralski in the recent past. The utility of coring rods perpendicularly to the growth axis will be a limiting factor for volume production. An ever increasing level of scrap will add to the cost of the substrates. While, if the results shown here for $4^{\prime \prime}$ boules can be scaled to $6^{\prime \prime}$ and $8^{\prime \prime}$ crystals, there is a strong logical argument that the Czochralski process can ultimately retake the role of the dominant production method for sapphire LED substrates. The Czochralski process is not without its own issues, not the least of which are overcoming the thermodynamic problems associated with melting large volumes of sapphire in graphite-heated systems or temperature limitations associated with iridium crucibles of large diameters.

\section{References}

[1] D.C. Harris, in: Proc. 10th DoD Electromagnetic Windows Symp., Norfolk, Virginia (USA), 2004.

[2] M.S. Akselrod, F.J. Bruni, J. Cryst. Growth 360, 134 (2012).

[3] C. Miyagawa, T. Kobayashi, T. Taishi, K. Hoshikawa, J. Cryst. Growth 372, 95 (2013).

[4] Commencement of Mass Production of Large Sapphire Substrates, www.ledinside.com/news/ 2012/11/sumitomo_metal_mining_large_sapphire_ substrates_20121116 (Accessed May 27, 2013).

[5] H.L. Tang, H.J. Li, J. Xu, in: Advanced Topics for Crystal Growth, Ed. S.O. Ferreira, 2013, p. 321.

[6] R.F. Belt, R.C. Puttbach, Mater. Res. Bull. 4, 403 (1969).

[7] V.S.F. Yip, C.D. Brandle, J. Am. Ceram. Soc. 61, 8 (1978).

[8] F. Degave, P. Ruterana, G. Nouet, J.H. Je, C.C. Kim, J. Phys. Condens. Matter 14, 13019 (2002).

[9] L. Liu, J.H. Edgar, Mater. Sci. Eng. R 37, 61 (2002).

[10] C.I.H. Asby, C.C. Mitchell, J. Han, N.A. Missert, P.P. Provencio, D.M. Follstaedt, G.M. Peake, L. Griego, Appl. Phys. Lett. 77, 3233 (2000).

[11] M. Gonsalves, W. Kim, V. Narayanan, S. Mahajan, J. Cryst. Growth 240, 347 (2002).

[12] M.J. Kappers, M.A. Moram, D.V. Sridhara Rao, C. McAleese, C.J. Humphreys, J. Cryst. Growth 312, 363 (2010).

[13] H.J.T. Ellingham, J. Soc. Chem. Ind. 63, 125 (1944).

[14] T.B. Reed, The Free Energy of Formation of Binary Compounds, MIT Press (1971). [Also available through the Biomass Energy Press, 2000.].

[15] F.D. Richardson, J.H.E. Jeffes, J. Iron Steel Inst. 160, 261 (1948).

[16] Yole Sapphire Market Forcast, www.i-micronews. com/upload/Rapports/Yole_Sapphire_Market November_2011_Flyer.pdf (Accessed May 28, 2013). 